\title{
Specifics in the review of health documentation
}

\author{
Kacalova $\mathrm{K}^{1}$, Havelkova $\mathrm{B}^{2}$, Farkasova $\mathrm{D}^{3}$ \\ The Slovak Medical University in Bratislava. Faculty of Nursing and Professional Health Studies. \\ Bratislava, Slovakia. katarina.kacalova@vszp.sk
}

\section{ABSTRACT}

OBJECTIVES: The objective of the work was to identify if health documentation management is depending of actually current legislation. Also to review range and consistency records. The work described forms and content of documentation, relating to treat wounds and decubitus.

METHODS: A content analysis of 139 health documentation of insurer of General Health Insurance Company, which were treated by 28 home care agencies (hereinafter "HCA") and in the HCA was done directly audit in 2012 and 2014. We set 29 assessment items, with criteria separation according structure, process and outcome. Documentation, we analyzed three ways: through 5 grade rating scale, by choosing a clear answer to the question: yes, no and evaluation of specific items of documentation.

RESULTS: The research confirm dependence keeping medical records in home nursing care agencies from legislation. On the other hand, it was found that the form of the recording method of health care in many cases are varied and fragmented. Deficiencies were found in the area of criteria such as structure, process and outcomes and transparency in the management of documentation pressure ulcers and chronic wounds. Only 62.9 $\%$ of the documentations was written nursing care and sent to Health Insurance Company in full compliance or with minor shortcomings.

CONCLUSION: Research has shown that in the legislation defined by standard forms was recorded the lowest number of deficiencies. This fact needs to be used for the development of new legislation, which directed to setting documentation with clearly defined, structured information's. WHO adopted the International Classification for Nursing Practice (ICNP) as essential and complementary part of professional health services (Tab. 2, Fig. 2, Ref. 22). Text in PDF www.elis.sk.

KEY WORDS: health documentation, legislation, Home care agency, health insurance

\section{Introduction}

Effectively setting the health care system in the current tendency to reduce the length of hospital stay, and appeals to the care of the chronically ill is a pan-European problem, which addresses health policy of individual states own and in cooperation. The development of medical knowledge, advent of new technologies and progress in the field of information, are leading to more accurate diagnosis and improving the quality of treatment, but also to the increase of the average age of the population and the need of solution of the providing non-hospital health care.

Almost all European countries are struggling with a lack of finance and professionally competent personnel for the care of the chronically ill in their natural social environment. Those aged 65 years or over will account for $29.5 \%$ of the EU-27's population by $2060(17.4 \%$ in 2010$)$. EU countries are also faced with the

${ }^{1}$ The Slovak Medical University in Bratislava. Faculty of Nursing and Professional Health Studies. Bratislava, Slovakia, ${ }^{2}$ General Health Insurance Company, directorate general, Bratislava, Slovakia, and ${ }^{3}$ The Slovak Medical University in Bratislava. Faculty of Nursing and Professional Health Studies, Bratislava, Slovakia

Address for correspondence: K. Kacalova, PhD, General Health Insurance Company, directorate general, Panonska cesta 2, SK-851 04 Bratislava, Slovakia.

Phone: +421.903 .605072$ problem of finding an integrated care system with sufficient flexibility to adapt to the changing needs of the people, while promoting the participation and decision-making (3).

Quality long-term care requires close cooperation between doctors and nurses, but also the patient himself and his relatives. Sharing of important information clear and understandable guidelines also require the accurate and clear record. Doctors and nurses have in institutional health care institutions, the opportunity to conduct the common chronological documentation. In the hospital was carried out a study aimed at monitoring compliance with the criteria structure of nursing documentation. Kilíková points to $94.6 \%$ meet the criteria of the structure standard in the results of monitoring, fulfillment the processual standard was in the range of $83.8 \%$ and the fulfillment of the criteria outcomes authors analyzed to $70.3 \%$. In one case it was found to record into documentation by unregistered nurse. In some documentations were missing a certain area of required data, the plan of nursing care was insufficient, the nursing process documentation was incomplete, unlimited access to the file, and have access to a documentation incompetent persons (6).

This monitoring points to elements differences and interdependencies of management of health records in institutional health care facility and HCA. The complexity of keeping health records is required in both types of healthcare facility. Registration nurses practicing the profession of nurse are required under 


\section{9-184}

current legislation. The possibility of peep into documentation is established by law and for the institutional and ambulant care is identical, but the conditions of ensuring for health records are not the same. Unlike hospital, in the HCA is health records, in most cases located at the place of business, where most often coming only appropriate personnel HCA.

The study carried out in Finland, the material was drawn from three databases: CINAHL, PubMed and Cochrane using the keywords nursing documentation, nursing care plan, nursing record system, evaluation and assessment. The search was confined to relevant electronically-retrievable studies published in the English language from 2000 to 2007. Studies classified under the heading of standardized documentation showed more positive than negative effects with respect to quality, the nursing process and terminology use, knowledge level and acceptance of computer use. In conclusion, it was found that the use of structured nursing terminology in electronic patient record systems will extend the scope of documentation research from assessing the quality of documentation to measuring patient outcomes (15).

In the ambulant area take care about long-term patients, in addition to ambulatory doctors and nurses, also home nursing agencies (hereinafter "HCA") that work in the branch autonomously. On a strategic position in the HCA system of hospital care also it highlights monograph titled Home nursing care (7). In doing so, close cooperation of doctors and nurses is needed. The proposal for the nursing care according to legal standards is mandatory part of medical records HCA. The proposal exposes and certifies attending physician and the professional representative HCA by their signature and stamp. This means that the responsibility lies with both sides. HCA nurses often treat patients with immobilization syndrome after stroke, after surgery, patients with compromised skin integrity and other health problems. Careful management of health records, which also includes a proposal for the nursing care, is in HCA necessary not only because of proper communication of information between nurses each other and in cooperation with the physician, but also like guidance in reimbursement of health care from insurance company.

The following research will focus on content analysis of documentation kept in HCA. The aim of the research was to determine whether the management of health records manages according to current applicable legislation. We watched consistency in the writing of health care in its various stages. Also, we investigated the presence and content of the additional forms documentation concerning the treatment of pressure ulcers and other non-healing wounds. In order to improve the quality of health care for the sick in their natural social environment cooperation it is essential not only to review nurses and nurses HCA, but, as well as mutual cooperation between nurses and doctors, whether in insurance company, or at the bedside. The common procedure for doctors and nurses while helping to gain the cooperation of the patient himself and his relatives, as in the case of long-term ill one of the most important factors in the success of health care.

\section{Materials and methods}

We chose the method of content analysis of health records HCA. We analyzed 139 randomly selected documentations insurers of General Health Insurance Company (hereinafter "GHIC"), treated through $28 \mathrm{HCA}$, where was made directly audit by review nurse in 2012 and 2014. Into research we have included documentation of insurers with sufficient data to analyze, especially of all documentation of insured persons, where were provided care for more than one month. In content analysis of nursing documentation we used Section 18-25 of Act no. 576/2004 Coll., Decree Ministry of Health guidelines on management of health documentation, nursing standards no. 2 Structure and management of nursing documentation, with determined by the current Professional Guideline of the Ministry of Health for the development, implementation and evaluation of standards, Ministry Decree no.364 / 2005, establishing the scope of nursing practice, the Ministry of Health Decree of the minimally requirements for personnel and material and technical provision health facilities and contracts with health insurance company $(11,12,4,5,13,1)$. We set 29 assessment items, with criteria separation according structure, process and outcome. Documentation, we analyzed three ways: through 5 grade rating scale, by choosing a clear answer to the question: yes, no and evaluation of specific items of documentation.

\section{The results of the research paper}

The first hypothesis examined mandatory forms of documentation (criteria of structure) and the identity of their use in individual HCA. In each HCA it was evaluated approximately 5 documentations recorded by nurses employed in the HCA. For statistical evaluation of each HCA alone was used chi-square test in contingency tables, the significance level $\alpha=0.05$. The results of tests aimed at detecting whether a particular HCA is governed by current legislation, which determines the presence of manda-

Tab. 1. Evaluation of the use forms provided by legislative of HCA (Criteria of structure - S1-S4).

\begin{tabular}{|c|c|c|c|c|c|}
\hline & \multirow{3}{*}{ Criterion } & \multicolumn{4}{|c|}{ Evaluation } \\
\hline & & \multicolumn{2}{|c|}{ Yes } & \multicolumn{2}{|c|}{ No } \\
\hline & & $\mathrm{n}$ & $\mathrm{f}$ & $\mathrm{n}$ & $\mathrm{f}$ \\
\hline $\mathrm{S} 1$ & 13. Does the documentation contain proposal for the nursing care? & 139 & $100 \%$ & 0 & $0 \%$ \\
\hline $\mathrm{S} 2$ & 14. Does the documentation contain arrangements for the provision of nursing care? & 131 & $94,2 \%$ & 8 & $5,8 \%$ \\
\hline S3 & 15. Does the documentation contain record of informed consent? & 96 & $69,1 \%$ & 43 & $30,9 \%$ \\
\hline S4 & 16. Does nurse record input history to currently valid record admission assessment of the patient? & 114 & $82,0 \%$ & 25 & $18,0 \%$ \\
\hline S5 & $\begin{array}{l}\text { 17. Does nurse record conducted nursing care in the nursing documentation, that is determining the } \\
\text { current Ministry of Health expert guidance about the management of medical documentation? }\end{array}$ & 80 & $57,5 \%$ & 59 & $42,5 \%$ \\
\hline
\end{tabular}


tory forms of documentation showed that in $46.4 \%$ (13 cases) HCA confirmed the statistical consistency of responses in $39.3 \%$ (11 cases) HCA was disapproved and $14.3 \%$ (4 cases) HCA has not been confirmed nor denied.

We then evaluated the answers to items $\breve{S} 1$ to $\breve{S} 5$ together in all HCA. The application form for the nursing care (the „Proposal“), received the most positive responses, was present in all surveyed documentation. On the other hand, most problems done recording of nursing healthcare into proper form courses of disease (Tab. 1).

Archiving of medical records is legislatively established for all types of health care providers. Is necessary also synoptically of the management of medical records, therefore was in HCA rated as one of the criteria results using 5-point scale assessment in defining the precise set of rules. In evaluation in a scale 1 (disagree) was identified for example documentation archiving by months when patients were treated, documentation wasn't conducted specifically for each patient. In assessment of the range of 2 (rather disagree) was identified documentation with missing records during the whole month, because documentation was produced by electronic system at the end of the month. With evaluation in the range of 3 (partially agree) was labeled documentation with lacking courses of disease, performances, were recorded in the protocol table of wounds, with irregular and hard to read records. Record of completed treatment patients was as a daily record in various forms - A4 sheet, workbook. Also documentation, which had identical records, no description was carried out for several months. Assessment of the range of 4 (previously agreed) was assigned documentation, where was difficult to search for information, documentation with lacking page numbering in the course of diseases, documentation with care planning in various places. Also documentation of inferior readability, where was into date box entered text, documentation with lacking record of year, there was recorded only day and month of treatment. Evaluation of transparency and the possibility of orienting in the nursing documentation pointed out some problems in some HCA. However, the final assessment is positive, which means that in most cases, ADOS maintain documentation transparently (range of $4+5=67.5 \%$ ).

Given that some HCA provide nursing care for a significantly larger number of patients compared to other (smaller) HCA, we watched consistency dependence fill in a form of documentation from the average number of treated patients, which was the objective of the second hypothesis. We divided the documentation into 2 groups according to whether the last two years were treated in HCA average less than 200 or more than 200 patients. We tested 16 items - selection process:

P1 Does input record nursing health assessment of the patient filled in completely and thoroughly? P2 Is nursing care plan (draft or separately) to the determination of individual nursing diagnoses? P3 Does the plan of nursing care contain all the necessary components (aims, nurse planned interventions, time element and frequency)?

P4 Is implemented nursing care recorded in the course of diseases according to the established plan of nursing care?

P5 Is recording courses of disease in clear, understandable, readable?
P6 Does record in documentation include description and performance evaluation?

P7 Does documentation contain for ongoing evaluation of nursing care that describe developments in the health status of the patient, the reprogramming of nursing care and consultation with a physician?

P8 Does each form of nursing documentation contain identification data indicated health care provider?

P9 Does each form of nursing documentation contain patient identifiers - name, surname and the patient's personal ID number?

P10 Are there in the documentation provided nursing diagnoses?

P11 Does documentation contain listed separately (except draft ...) nursing plan made on the basis of nursing diagnosis defined at time of admission to the HCA?

P12 Does courses of disease contain: P12a) date, P12b) time, $\mathrm{P} 12 \mathrm{c}$ ) the signature of nurse / health professional who realized performance?

P13 Is the signature of the nurse readable?

P14 Is signing nurse stamp located with the name?

P15 Are there specific forms filled out properly?

P16 Are there specific forms of nursing documentation marked with the identification data of the health care providers, name and surname of the patient, his birth number? For statistical analysis, we used the chi-square test and Fisher's test.

The results show that $50 \%$ of test items have been consistently held in the HCA with a greater average number of treated patients and $43.75 \%$ wasn't statistically significant difference. Only one item relating to the presence of continuous assessment of health care came out the opposite. Result, it was found that HCA with

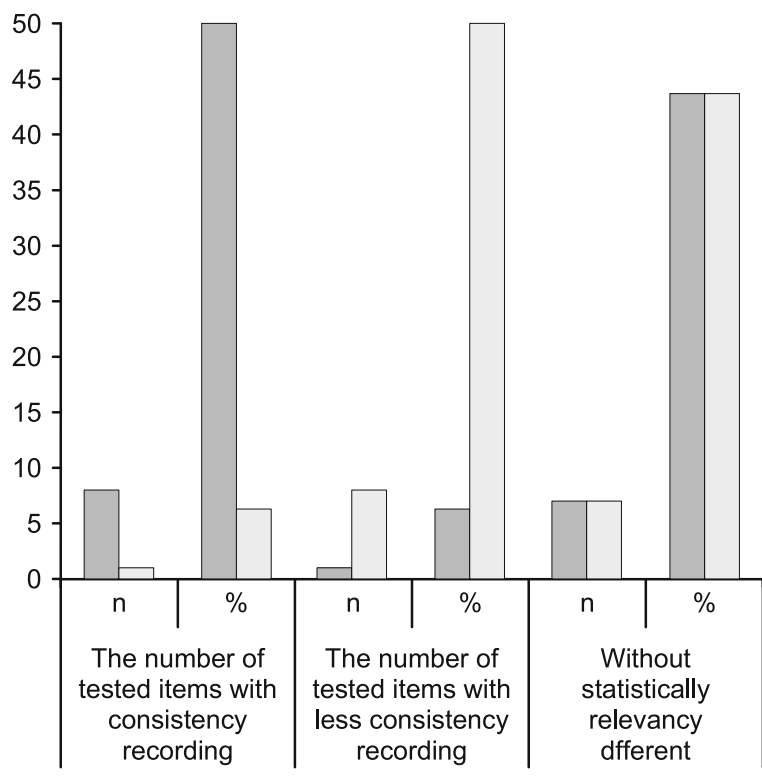

$\square$ HCA with greater average of number treated patient

HCA with less average of number treated patient

Fig. 1. Consistency of recording data in the dossier. 


\section{9-184}

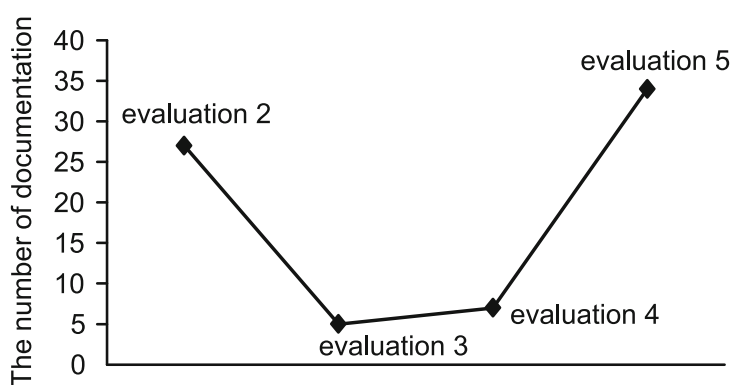

Fig. 2. Evaluation of thoroughness of recording nursing care to specific forms.

a smaller number of patients treated do not manage documentation more consistently as documentation HCA with most of the treated patients (Fig. 1).

When analyzing, we also evaluated the compliance of health care recognized in the invoice to the fixation of health care in the patient documentation with a 5-point scale with responses with the rules of evaluation. The results were then evaluated and percentages. In evaluation in a scale 1 (disagree) was identified for example documentation, where was nursing care unrecorded or recognized during hospitalization, or documentation, where was other recorded performances than reported. In assessment of the range of 2 (rather disagree) was identified documentation, where has not been recorded more than one of the declared performances. With evaluation in the range of 3 (partially agree) was labeled documentation, in which has not been recorded one of a number of recognized performances. Assessment of range of 4 (previously agreed) was assigned documentation, where was occurrence of duplication of performances along with other expertise. In range of 5 (fully agreed) was assigned record of nursing care, which was fully compliant. Results showed that $51.08 \%$ (71) $100 \%$ (139) of the dossiers had been properly registered nursing care recognized health insurance company. We met with the documentation, which were recorded performances during hospitalization or interruption of care, meaning that they were not carried through HCA. In some dossiers lacked recording physiological functions, continuous evaluation of wounds, record about consultation with a doctor, and these performances were reported to insurance company.

Content analysis was, in the hypotheses number three, detected the presence of specific forms of wound care in health records and found that most often lead HCA form of wound care protocol in $62.4 \%$.

Evaluation of consistency record of health care into specific forms pointed to a surprisingly high number of conflicting evaluation results (3) (Fig. 2).

\section{Discussion}

The research confirmed dependence keeping medical records in the HCA from current legislation. On the other hand, it was found that the form and method of recording health care are inconsistent in many HCA. The problem is often the reluctance of physicians to cooperate with the HCA (18). Also, their lack of awareness about nursing interventions codes listed not only in the proposal and medical records but also is sent Health Insurance Company for reimbursement. It happens that the nurses filled out the form alone, but without sufficient information on the case history health status and medical diagnosis. The problem resides in the lack of awareness of doctor about health immobile patients after discharge from the hospital, particularly its skin's deficits, which are not often in the medical final report (2).

A systematic review of the literature relating to the monitoring of the recording of the nursing process in medical records was conducted in one of the Swiss study. There were monitored the accuracy, completeness and consistency of nursing diagnoses, interventions and outcomes. The result was the detection of irregularities in diagnostic accuracy and recording symptoms and etiologies. Despite the variable resulting findings of the study points to a trend in which the nursing diagnostics to improve the evaluation of nursing documentation, quality of the recorded interventions and achieved results (9).

Monitoring of articles relating to the evaluation of nursing documentation in the years 2000-2010, it was found that the quality of the documentation was measured using a variety of auditing tools using both qualitative and quantitative evaluation. Documentation was evaluated regarding structure and format, process and content. Weaknesses were identified in documentation and study information the impact on its quality. In conclusion, it was stated that research should pay more attention to the accuracy of nursing documentation, further problems that lead to variations recorded, lack of quality documentation and finally the effects of these factors on nursing practice and the results achieved by the patient (20).

The increasing average age, with the progress in medicine, technology and other related fields heading to the increasing need to ensure long-term care for the chronically ill. GHIC carried out analysis and the results suggest that HCA treat an increasing number of insurers (Tab. 2).

This is closely related the need and development in the field of nursing and ensuring reliable cooperation doctors and nurses. Proper written proposals for the provision of nursing care, provision and reporting of health care and management medical documentation in accordance with applicable legislation leads to improving the quality of care while also increasing the benefit to the patient. Practice often brings results reflecting improved health for patients, where was really corroborated documented close cooperation between doctors and nurses and vice versa worsening or persistence of health problems in patients in who medical care not striking a balance between medical and nursing options.

Tab. 2. The number insurers of General Health Insurance Company treated by HCA (19).

\begin{tabular}{lc}
\hline Year & Number of treated patient by HCA \\
\hline 2010 & 26265 \\
2011 & 28539 \\
2012 & 30663 \\
2013 & 32836 \\
2014 & 33891 \\
\hline
\end{tabular}


Ondruš points out that "incorrect treatment of chronic diseases can now lead to a $70 \%$ increase in the cost of health care" (10). As an example, the health care of patients with pressure ulcers and non-healing chronic wounds, where a need for cooperation of all three is parties - the patient (relatives), medical doctors and nurses. Patients and their relatives often gather information about their disease and its treatment of social media. The need for cooperation between patient and health professionals and described in several international studies.

One study from Netherlands devoted systematic evaluation of the literature dedicated effects of using social media by patients. It was found that social media information can on the one hand to bring benefit to patients, on the other; they may be harmful to provide information. Of the selected 22 articles included in the study were identified several categories of uses and types of effects. Positive effects are for example improved self- management and control, enhanced psychological well-being, negative effects can be diminished subjective well-being and loss of privacy. "Social media use by patients was found to affect the healthcare professional and patient relationship, by leading to more equal communication between the patient and healthcare professional. On the other hand, "healthcare professional bears full responsibility for the decision taken. When patients bring in the information from social media to the consultation, this could lead to unnecessary processes of sorting relevant information from irrelevant information." (17) Such a situation does not always positively affect the relationship healthcare professional and patient.

The complexity and also the need for mutual communication between patients, doctor and nurse also points out another English observational study about a mismatch between population health literacy and complexity of health information. "Sixty-four health materials were sampled. All contained literacy (text) information, and 50 also contained numeracy information. Of the 5795 participants who had completed the literacy skills tests, 2515 (43\%) were below the text-only competency threshold, while of the 4767 people who had completed both the literacy and numeracy skills assessments, $2905(61 \%)$ were below the literacy + numeracy competency threshold." The results of studies show that health materials are too complex for the skills of a significant proportion of the population, resulting in less access to health information. General practitioners (GPs) have a key role through patient care and through their roles as health service commissioners. (14)

GPs in Slovakia have possibility to indicate of nursing care for a seriously ill through HCA. Nurses from HCA in practice regularly instruct relatives or community members and do professional training required performance when attending patients in the household within the medical treatments. These activities is paid by health insurance company and creates a formal space for proper communication and guidance to patients directly in their natural social environment, taking into account their individual needs and possible additional consultation with a doctor.

A separate issue concerning not only written communications between healthcare professionals is also a topic of palliative care and its documentation. A systematic review called Evidence still insufficient that advance care documentation (ACD) leads to en- gagement of healthcare professionals in end-of-life discussions. It describes the meaning of ACD. Although the research assumption wasn't confirmed in all available English speaking studies, its practical significance is growing. A total of 24 studies involving 23,914 patients bereaved family members, doctors, nurses, community care staff, government policy-makers and judges met the criteria for inclusion. "The studies largely (18/24) found staff had positive attitudes towards the use of advance care planning documents as instruments to improve communications with patients and families, and encouraged or facilitated future care planning." The study points out, that "conversations may be easier to initiate in palliative units or nursing homes where death is expected and there is time for discussions, compared to critical and general hospital wards". The authors point out that "end-of-life conversations can be challenging and rewarding for doctors and patients. (8)

Similarly, another, Norwegian research project concerns seriously ill patients. It targeted to reducing risk and enhances safety for patients who want to combine conventional medicine with complementary and alternative medicine (CAM) in cancer care. This cross-sectional protocol states, that the majority of patients with cancer use CAM because they believe it increases the body's ability to fight the cancer. Healthcare providers should assist patients in safeguarding their treatment decision. The problem is, that the current non-communication between CAM and conventional providers leaves it up to the patients themselves to choose how to best integrate the two worlds of therapy." However, the patient may thereby be endangered. Direct risk is caused by the treatment itself(for example: bleeding in response to acupuncture needling, adverse effect of an herb). Indirect risk is related to adverse effects of the treatment context, for example, the CAM practitioner rather than the medicine. A patient may be harmed by a care context that prevents the patient from receiving the best possible treatment relevant to her or his health needs. (16)

From previous articles follows that the weak situation in the difficult treatment of severe diseases is resolved not only health professionals but also patients themselves or their relatives. All three parties are trying to find a way out of a difficult situation suffered. Patients often on social networks or in alternative medicine, health care workers with the strain of our endeavors to assist in their professional capacity, which may lead to tension and exhaustion on both sides. The following study aims to help in such situations, but not in terms of medicine or alternative treatments but from the perspective of the impact of arts in healthcare facilities.

English authors of the study conducted a critical review of the literature between 2004 and 2014. There were 27 articles reviewed. "Arts interventions were diverse and included music listening, visual arts, reading and creative writing, and dance. I was found that "introducing arts activities into a variety of different healthcare settings can have a positive impact on the health and well-being of patients and service users, and healthcare professionals hold predominantly positive perceptions of their use." For example, music "improved mood and reduced stress for patients, produced a sense of familiarity in a strange environment for patients, improved sleep for patient, improved communication, mood and psychological well-being." (22) 


\section{Conclusion}

A medical record to some extent reflects the character of work and the specific character of healthcare employer. It is necessary to update the legislation and to further specify the necessary and practical part of the documentation used. Also, monitor developments in the recording of health care in the documentation, which affects not only the education, revision control, medical progress, as well as possibility to take forward information technology. Just the information technologies enable more effectiveness not only to the actual recording documentation healthcare, finding the necessary data for evaluation but also to improve communication between health care professionals. The research results also indicate the need to establish a uniform structure and format for the recording. Structured information would allow subsequent comparison of documentation in the medicine branch, and the results of comparison could lead to important finding's for future patient care. Besides, the research results point on the need for recording even other forms of care, which directly or indirectly affect the health of the patient and its further development. These records may, as valuable sources of information, significantly help healthcare professionals to care for a seriously ill in the future. In Slovakia, it is currently developing the issue of palliative medicine, where have their irreplaceable place doctors and nurses. Health insurance companies have contract with the hospices, mobile hospices, and at present, the contractual relationship extends to ambulance of palliative medicine. Under certain conditions, severely ill patients can to spend the last moments of life in their home environment. It requires close cooperation relatives, nurses and doctors and taking care of properly recorded documentation, which becomes the guiding factor for further decisions not only on the individual patient, but documentation becomes a source of valuable information and knowledge for future generations.

\section{References}

1. Act. no. 576/2004 Coll. on healthcare-related services, health care and on amendments to certain laws.

2. Bušová B. Articles about nursing. Bratislava: Harris Slovakia, 2013.

3. European Quality Framework for long-term care services. Why a European Quality Framework for long-term care services? 2012. In WEDO: For the wellbeing and Dignity of Older people. 2012, $48 \mathrm{~s}$.

4. http:// wedo.tttp.eu/system/files/24171_WeDo_brochure_A4_48p_ EN_WEB.pdf.

5. Health Ministry Decree no. 306/2005 Coll., Establishing a list of nursing diagnoses

6. Health Ministry Decree no. 364/2005 Coll., establishing the scope of nursing practice provided by the nurse independently and in collaboration with the physician and the scope of midwifery practice provided by the midwife independently and in cooperation with the physician.

7. Kilíková M. Quality management documentation in nursing practice. Kontakt 2009; 11 (1): 270.

8. Kožuchová M et al. Home nursing care. Martin: Osveta, 2014, 244 s.

9. Lewis E, Cardona-Morrell M, Ong KY, Trankle SA, Hillman K.

Evidence still insufficient that advance care documentation leads to en- gagement of healthcare professionals in end-of-life discussions: A systematic review. Sage J 2016; 30 (9): 807-824. http://pmj.sagepub.com/ content/30/9/807.abstract

10. Müller-Staub MM, Lavin MA, Needham I, Achterberg TV. Nursing diagnoses, interventions and outcomes - application and impact on nursing practice: systematic review. J Adv Nursing 2007; 56 (5). http:// onlinelibrary.wiley.com/journal/10.1111/(ISSN)1365-2648/issues.

11. Ondruš P. World health systems in the face of globalization. Banská Bystrica: PRO, 2014, 319 s.

12. Professional guidance to the Ministry of Health no. 16138-2 / 2004OO implementation for the development and evaluation of standards in nursing and midwifery. Official in the Ministry of Health. 2004 yer.52, special edition.

13. Professional guidance to the Ministry of Health no. 07594/2009CFC management of medical records. Official in the Ministry of Health. 2009 year.57, item $42-48$.

14. Rescript Ministry of Health no. 09812/2008-OL of 10 September 2008 concerning minimum requirements for personal and material and technical equipment of various types of healthcare facilities. Gazette Min Health 2008; 56, item 32-51.

15. Rowlands G, Protheroe J, Winkley J, Richardson M, Seed PT, Rudd R. A mismatch between population health literacy and the complexity of health information: an observational study. Brit J Gen Practice 2015; 65 (635): e379-e386. http://bjgp.org/content/65/635/e37921.

16. Saranto K, Kinnunen UM. Evaluating nursing documentation - research designs and methods: systematic review. J Adv Nursing 2009, 65 (3). http://onlinelibrary.wiley.com/journal/10.1111/(ISSN)1365-2648/issues. ISSN 13652648.

17. Stub T, Musial F, Quandt S, Arcury T, Salamonsen A, Kristoffersen A, Berntsen G. Mapping the risk perception and communication gap between different professions of healthcare providers in cancer care: a cross-sectional protocol. BMJ 2015; 5 (e 008236). http://bmjopen.bmj.com/ content/5/9/e008236.full?sid=14a6148c-cbe5-4caf-aca4-b4629cf75146.

18. Smailhodzic E, Hooijsma W, Boonstra A, Langley DJ. Social media use in healthcare: A systematic review of effects on patients and on their relationship with healthcare professionals. Health Serv Res Sseries 2016. http://bmchealthservres.biomedcentral.com/articles/10.1186/s12913016-1691-0

19. Tabaková M. The principle of justice in the nursing home. Nursing Midwifery 2006; 8 (2): 257-264.

20. The number patient of General Health Insurance Company (GHIC), whou had a nurse care. Analysis GHIC 2014.

21. Wang N, Hailey D, Yu P. Quality of nursing documentation and approaches to its evaluation: a mixed-method systematic review. J Adv Nursing 2011; 67 (9). http://onlinelibrary.wiley.com/doi/10.1111/jan.2011.67. issue-9/issuetoc.

22. World Health Organization. International Classification for Nursing Practice (ICNP). 2016. [online] http://www.who.int/classifications/ icd/adaptations/icnp/en/.

23. Wilson C, Bungay H, Munn-Giddings C, Boyce M. Healthcare professionals' perceptions of the value and impact of the arts in healthcare settings: A critical review of the literature. Internat J Nursing Stud 2016; 56 (0020-7489). doi.org/10.1016/j.ijnurstu.2015.11.003.

Received September 28, 2016. Accepted November 30, 2016. 